\title{
Effect of the Graphene Sheets on the Physical Properties of Copper Sulfide Nanoparticles
}

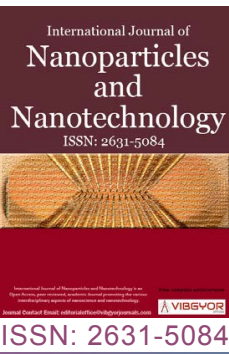

\section{Al Alwani Ammar J", Pandey Shipra, Pandey Shiv, Kletsov Alexey A, SB Venig and Glukhovskoy Evgeny $G$}

Saratov State University, Saratov, Russia

\begin{abstract}
This paper shows the synthesis of copper sulfide (CuS) nanoparticle by co-perception method at low temperature and the synthesis of copper sulfide/graphene sheets (CuS/Gs) nanocomposite by hydrothermal method at $120^{\circ} \mathrm{C}$. The structure, surface morphology and size of nanoparticles (NPs) are discussed and analyzed by X-ray diffraction analysis (XRD), energy dispersive X-ray analysis (EDX) and transmission electron microscopy (TEM). The obtained nanoparticles have sizes between $(4-10 \mathrm{~nm})$. We are studied the effect of graphene-based nanoparticles nanocomposite on the thermal properties by thermogravimetric analysis (TGA). The photocatalysis properties are investigated at different conditions. The result shows that the graphene sheet (Gs) nanocomposite effect the composition and the thermal properties of nanoparticles.
\end{abstract}

\section{Keywords}

Hydrothermal, Nanoparticle, Graphene sheets, Nanocomposite, Thermogravimetric analysis

\section{Introduction}

Copper sulfide (CUS) nanoparticles are widely used in various applications of different fields such as $p$-type semiconductor, cathode materials in high capacity secondary type lithium batteries and other applications [1-6] due to their potential importance for application and fundamental scientific research.

At present, the transition semiconductor NPs adoption of chalcogenide material is interesting too because of the physical and chemical properties of the unusual, including electrical conductivity, and the ability to chemical sensing properties ideal for solar energy conversion [7-10].

The copper sulfide nanocrystals with various morphologies (nanoparticles, nanorods and nanotubes) have been prepared by various methods such as microwave-assisted methods [11], electrosynthesis [12], solvothermal process [13], solid-state reactions [14], mechanochemical route [15] and chemical vapor deposition (CVD) [16]. Most of these methods need special instruments, high temperature, inert gas environment, and long reaction time.

The more simple routes and the cheap materials have been used to fabricate copper sulfide NPs.

*Corresponding author: Al Alwani Ammar J, Saratov State University, Astrakhanskaya, 83, Saratov, 410012, Russia

Accepted: June 13, 2019; Published: June 15, 2019

Copyright: (c) 2019 Al Alwani AJ, et al. This is an open-access article distributed under the terms of the Creative Commons Attribution License, which permits unrestricted use, distribution, and reproduction in any medium, provided the original author and source are credited.

Al Alwani et al. Int J Nanoparticles Nanotech 2019, 5:029 
Co-precipitation method of preparing CUS nanoparticles is technologically convenient and ecologically safe for the environment. We have studied the thermal properties of CuS and CuS/Gs nanocomposite. Our results have been showed that the synthesized CuS/Gs nanocomposites have controllable size, the morphology more homogeneous and enhanced the thermal properties.

\section{Material and Methods}

\section{Preparation of copper sulfide}

The synthesis of copper sulfide nanoparticles at low temperature was carried by co-precipitation method. The NPs were prepared in solution using the starting materials ionic copper (II) chloride dihydrate $\left(\mathrm{CuCl}_{2} \cdot 2 \mathrm{H}_{2} \mathrm{O}\right)$ and thiourea $\left(\mathrm{CH}_{4} \mathrm{~N}_{2} \mathrm{~S}\right)$ in the presence of dextrose. In this method reaction is carried out in ethanol, distilled water acts as a mild fuel and help stimulates the reaction with sufficient activation energy at a lower temperature. Copper (II) chloride dihydrate (dried powder) was dissolved in 60:40 ratio solvent mixture of ethanol: Distilled water to a concentration of $0.1 \mathrm{M}$ and stirring for 30 minutes at room temperature. $0.5 \mathrm{~g}$ dextrose was added to the solution, the final $\mathrm{pH}$ of the solution is maintained to 9.0 using $10 \mathrm{mM}$ sodium hydroxide $(\mathrm{NaOH})$. The prepared solution was subjected to continuous stirring for $30 \mathrm{~min}$ at of $70^{\circ} \mathrm{C}$. Thiourea $\left(\mathrm{CH}_{4} \mathrm{~N}_{2} \mathrm{~S}\right)$ is dissolved in ethanol (concentration $0.1 \mathrm{M})$, then the solution was added dropwise to the final solution and stirred for 2 hours with continuous heating at $70{ }^{\circ} \mathrm{C}$. The powder obtained was washed with ethanol, distilled water four times to remove the uncapped dextrose and finally with ethanol and dried at ambience.

\section{Preparation of copper sulfide/graphene sheets (CuS/Gs)}

(0.2986 g) of copper (II) chloride dihydrate is dissolved in $12 \mathrm{ml}$ of ethanol:distilled water mixture ratio (60:40) respectively and stirring $30 \mathrm{~min}, 0.4$ $\mathrm{g}$ of dextrose was added and the final $\mathrm{pH}$ of the solution is maintained to 9.0 by using $10 \mathrm{mM}$ sodium hydroxide $(\mathrm{NaOH})$. The prepared solution was stirred for $30 \mathrm{~min}$ at of $70^{\circ} \mathrm{C}$.

$0.008 \mathrm{~g}$ Gs solution dissolved in $2 \mathrm{ml}$ ethanol and sonicated then added to the solution above.

Another container $0.11418 \mathrm{~g}$ thiourea was dissolved in $12 \mathrm{ml}$ ethanol and added dropwise to the solution above with stirring and heating at $70{ }^{\circ} \mathrm{C}$, maintain $\mathrm{pH}$ by $\mathrm{NaOH}$, the final solution further stirred for $2 \mathrm{~h}$ with continuous heating at $70{ }^{\circ} \mathrm{C}$. Then the final solution placed in hydrothermal vessel for $6 \mathrm{~h}$ at $120^{\circ} \mathrm{C}$, after cooling the vessel washed with ethanol and distilled water four times to remove the uncapped dextrose and finally with ethanol and dried at ambience.

\section{Results and Discussion}

\section{X-Ray diffraction of copper sulfide}

Figure 1 shows the XRD patterns of synthesized copper sulfide NPs. Samples are well crystallized. All primary diffraction peaks of the curve are in good agreement with the standard data of CuS (JCPDS № 06-0464). There are peaks with $2 \theta$ values of $\left(29.354^{\circ}, 31.990^{\circ}, 39.000^{\circ}, 47.799^{\circ}, 53.024^{\circ}\right.$, $56.104^{\circ}, 58.716^{\circ}$ ) corresponding to copper sulfide nanoparticles crystal planes of the preferential orientation (102), (103), (105), (110), (108) , (202), (116) respectively and are in good agreement with the pattern (JCPDS № 06-464). The most intense peak at $2 \theta=31.990$ relates to (103). The other peaks seen on the XRD scan have low intensities and full-width half-maximum (FWHM). The copper sulfide particles have a cubic structure.

The XRD pattern demonstrates that the products grown under low temperature are copper sulfide NPs with cubic structure, the obtained diffraction peaks broadened by the small diameter of the

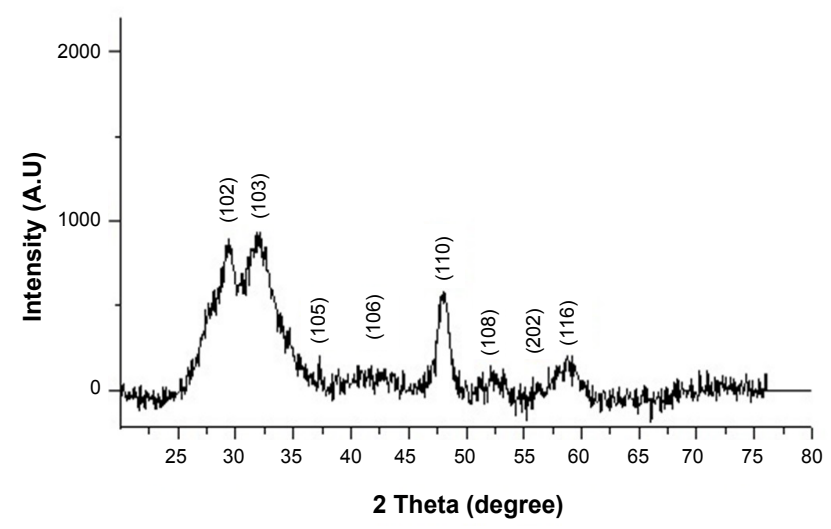

Figure 1: XRD of copper sulfide nanoparticles.

Table 1: XRD data of copper sulfide.

\begin{tabular}{|l|l|l|l|l|}
\hline $\begin{array}{l}\text { Miller's index } \\
\text { (lattice plane) }\end{array}$ & $-\mathbf{1 0 2}$ & -103 & -110 & -116 \\
\hline$\beta$ & $2.530^{\circ}$ & $3.54^{\circ}$ & $1.024^{\circ}$ & $1.517^{\circ}$ \\
\hline $2 \theta$ & $29.35^{\circ}$ & $31.99^{\circ}$ & $47.79^{\circ}$ & $58.71^{\circ}$ \\
\hline Grain size (g), nm & 5.5 & 4 & 14 & 10.3 \\
\hline
\end{tabular}


nanoparticles. To calculate the size of nanoparticles used Scherrer equation:

$$
g=\frac{0.89 \lambda}{\beta \cos \theta}
$$

Values of the grain size $(g)$, fullwidth at half maximum ( $(B)$, wavelength $(\lambda)$ and diffraction angles $(\theta)$. Miler's index (lattice plane) are summarized in the following (Table 1):

\section{Transmission electron microscope (TEM)}

Figure 2 shown the TEM images of copper sulfide nanoparticles produced at a temperature of $70{ }^{\circ} \mathrm{C}$ for $2 \mathrm{~h}$. It can be observed that the sample appears to have a dimension of $6 \mathrm{~nm}$ and $11 \mathrm{~nm}$, and weak agglomeration because of physical and chemical properties of the two metals, the TEM image that the average nanoparticles size is prox- imally $9 \mathrm{~nm}$, which is in agreement with the size obtained from XRD of copper sulfide NPs.

\section{Energy dispersive X-ray analysis (EDX)}

Figure 3a shows the energy dispersive X-ray analysis (EDX) of CuS that the required phase of copper $(\mathrm{Cu})$ and Sulfide $(\mathrm{S})$ is present in the sample. The graph also shows the presence of carbon (C) and oxygen $(O)$ in the EDX picture of copper sulfide NPs. The chemical compositions and mineral phases of each sample are approved. Figure $3 b$ shows the energy dispersive x-ray analysis (EDX) of CuS/Gs nanocomposite proved that the required phase of copper ( $\mathrm{Cu}$ ) and sulfide (S) is present in the sample also. The graph shows the presence of carbon (C) and oxygen (O) are present in the EDX picture of copper sulfide/graphene sheets.
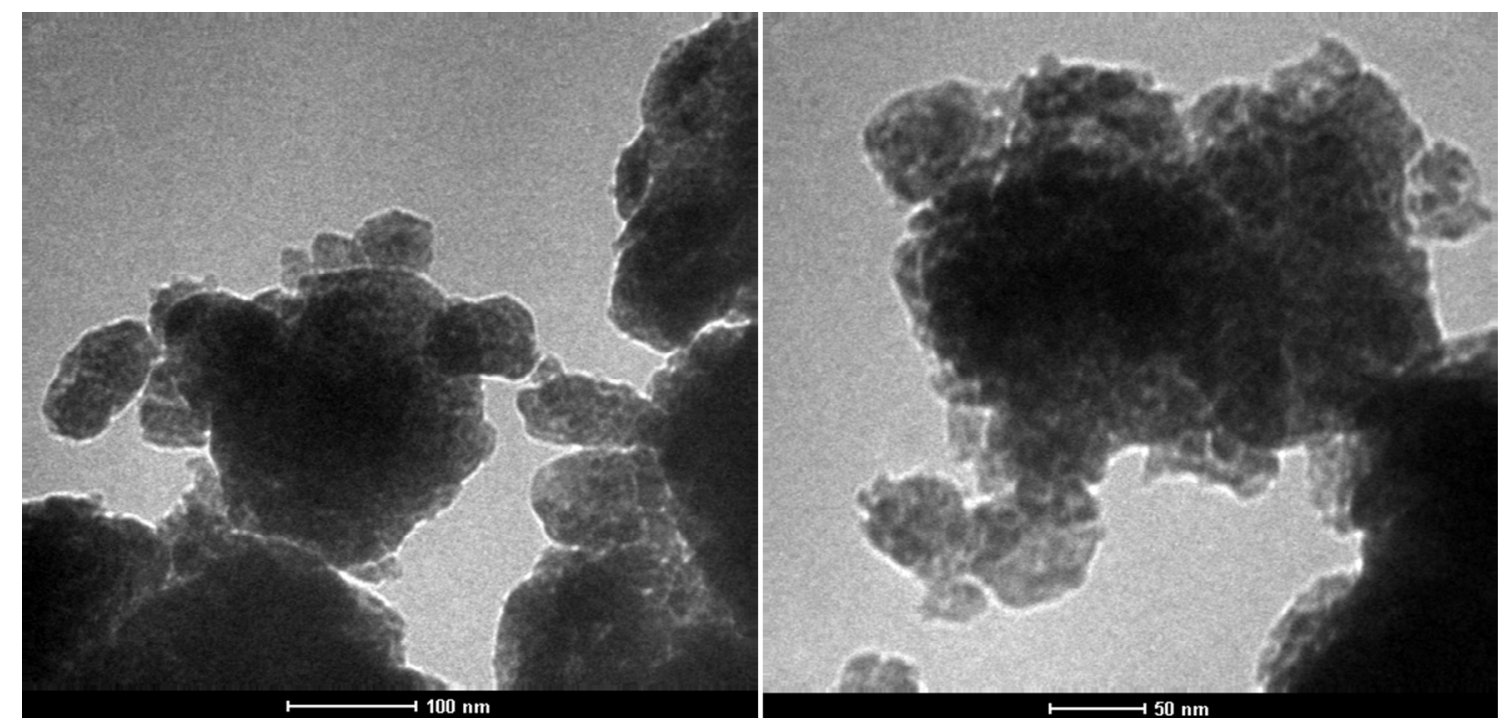

Figure 2: TEM Images of copper sulfide nanoparticles.
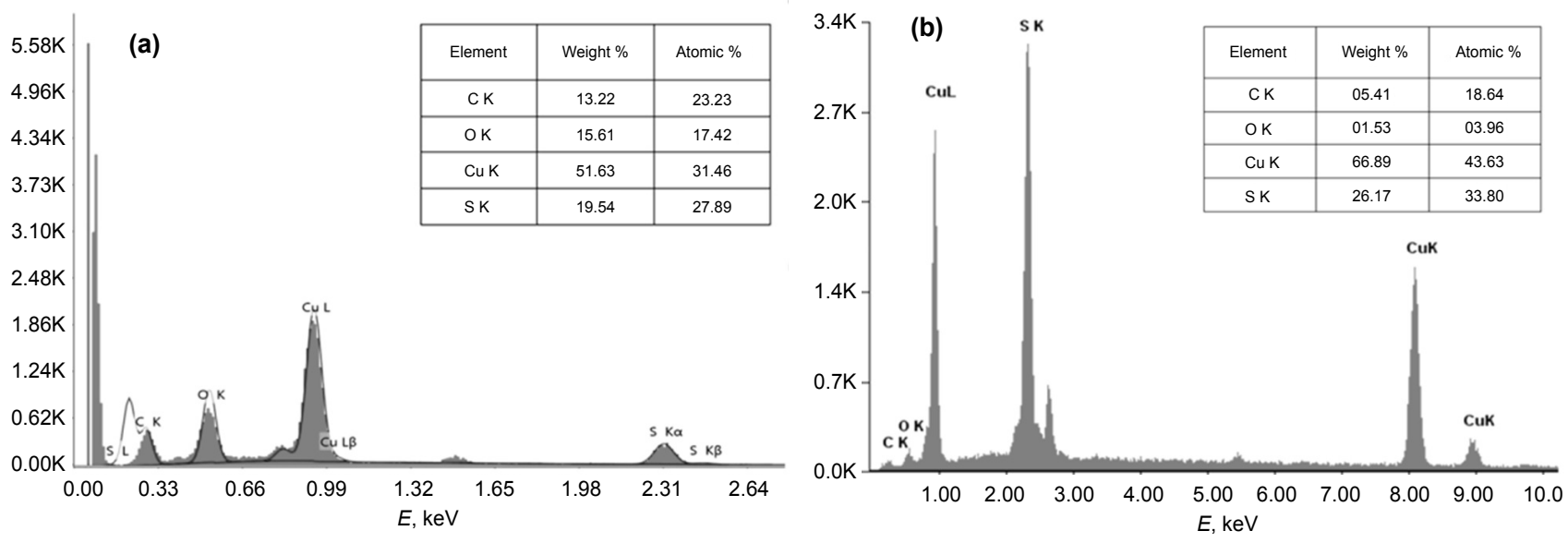

Figure 3: The energy dispersive X-ray analysis (EDX) of CuS (a), CuS/Gs (b). 


\section{Thermogravimetric analysis of copper sulfide}

The thermal degradation, impurity concentration and phase change of copper sulfide have been studied under various temperature program of $50^{\circ}$ $900{ }^{\circ} \mathrm{C}$ at a scan rate of $10^{\circ} \mathrm{C} / \mathrm{min}$ in the $\mathrm{N}_{2}$ atmosphere.

A quantitative thermogram of CuS has shown four weight losses with a total degradation of $44.04 \%$. The step 1 degradation between the temperatures of $50^{\circ}-140{ }^{\circ} \mathrm{C}$ has shown $9.94 \%$ weight loss that is attributed to the moisture content in the sample. A substantial degradation of $23.0 \%$ around $170^{\circ}-380^{\circ} \mathrm{C}$ has been associated with removal of oxygenated entities and the impurities related to the solvents or its bi-products. A decomposition of $8.2 \%$ between the temperatures of 387 ${ }^{\circ} \mathrm{C}-480{ }^{\circ} \mathrm{C}$ is accompanied by removal of residual amorphous carbon contents remained with the copper sulfide even after washing. A mild degradation of $2.9 \%$ is observed in a broad temperature range of $650^{\circ}-713^{\circ} \mathrm{C}$ that is attributed to the phase change in CuS. Furthermore, no any other weight loss is observed in the remaining temperature range up to $900{ }^{\circ} \mathrm{C}$. The corresponding differential scanning calorimetric (DSC) have shown endothermic reaction coordinate for removal of moisture contents while exothermic for the elimination of oxygen related moieties, phase change and the oxidation of carbon contents into $\mathrm{CO}_{2}$.

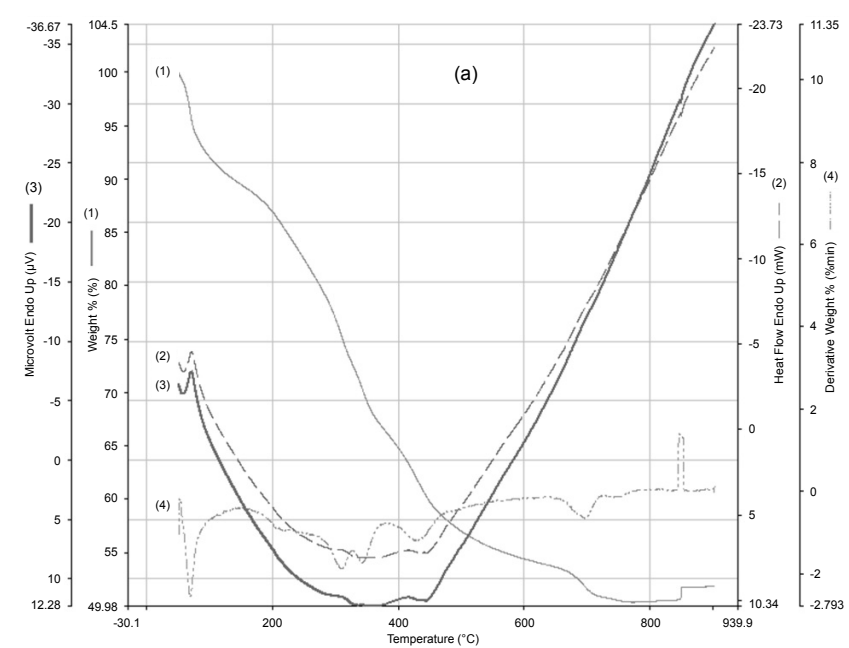

Figure $4 a$ of copper sulfide NPs shown thermogravimetric analysis (TGA) and derivative thermogravimetry (DTG) curves have shown four-step degradation process; one associated with the elimination of moisture contents while other with the ionization of oxygen individuals, residual amorphous carbon contents, phase change and removal of associated impurities. Differential scanning calorimetric (DSC) analysis has shown the endothermic and exothermic enthalpy change during the alleviation of copper sulfide impurities.

Figure 4 a of copper sulfide NPs shows differential scanning calorimetric (DSC) curve corresponding to TGA curves in certain temperature ranges revealed exothermic peak at $438^{\circ} \mathrm{C}$. The first transition corresponding to the second weight loss occurs at $68^{\circ} \mathrm{C}$, the third transition corresponding to weight loss at $445^{\circ} \mathrm{C}$.

\section{Thermogravimetric analysis of copper sulfide/ graphene sheets composite}

The thermal degradation, impurity concentration and phase change of copper sulfide/graphene sheet have been studied under the temperature program of $50^{\circ}-900^{\circ} \mathrm{C}$ at the scan rate of $10^{\circ} \mathrm{C} / \mathrm{min}$ in the $\mathrm{N}_{2}$ atmosphere. A quantitative thermogram of CuS/Gs has shown four weight losses with a total degradation of $37.68 \%$. The loss weight of $(6.6 \%)$ in the region between $54{ }^{\circ} \mathrm{C}-203{ }^{\circ} \mathrm{C}$ that is attributed to the moisture content in the sample, substan-

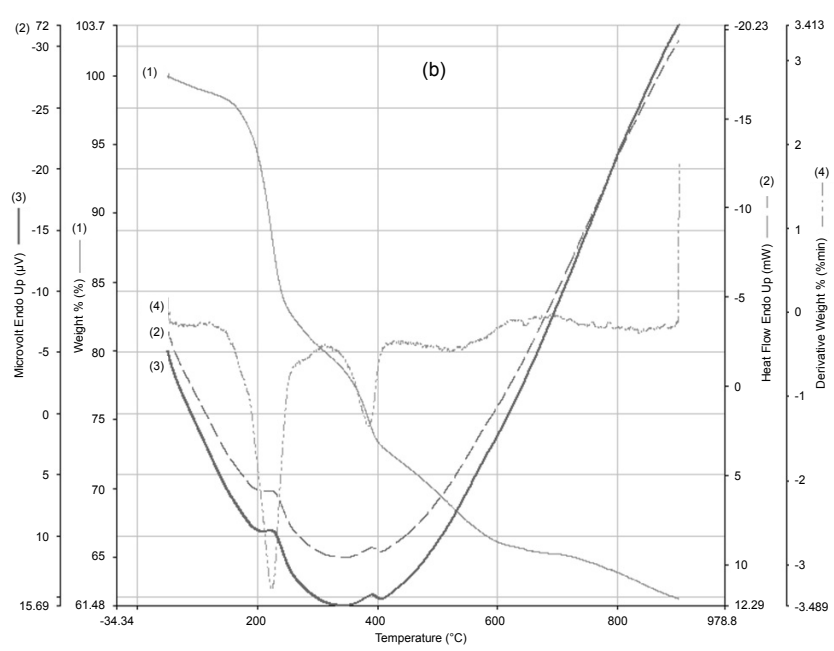

Figure 4: Thermogravimetric of CuS NPs a) CuS/Gs; b) TGA curve 1), DTG curve; 4) Have shown four-step degradation process; one associated with elimination of moisture contents while other with the ionization of oxygen individuals, residual amorphous carbon contents, phase change and removal of associated impurities. DSC (2) Analysis has shown the endothermic and exothermic enthalpy change during the alleviation of copper sulfide and copper sulfide/graphene sheets impurities, DTA (3). 
tial degradation of $(21.56 \%)$ in the region $228{ }^{\circ} \mathrm{C}$ and $345^{\circ} \mathrm{C}$ due to decomposition of dextrose. The third transition between $360.91^{\circ} \mathrm{C}$ and $515.12{ }^{\circ} \mathrm{C}$ a weight loss of $30.9 \%$, the fourth decomposition between $718.46{ }^{\circ} \mathrm{C}-891.24{ }^{\circ} \mathrm{C}$ weight losses (37.95\%).

Figure $4 \mathrm{~b}$ of copper sulfide/graphene sheets shows differential scanning calorimetric (DSC) curves corresponding to thermogravimetric analysis (TGA) curves in certain temperature ranges revealed endothermic reaction coordinate for removal of moisture contents while the exothermic peak at $410{ }^{\circ} \mathrm{C}$ for the elimination of oxygen related moieties. The first transition, corresponding to the second weight loss, occurs at $68^{\circ} \mathrm{C}$, the third transition corresponding to weight loss at $440{ }^{\circ} \mathrm{C}$.

In the (Figure $4 \mathrm{~b}$ ) we observed the maximum peak temperatures detected on the derivative thermogravimetry (DTG) curve, which depends on the heating rate, the maximum temperature of 223 ${ }^{\circ} \mathrm{C}$ is due to the decomposition this is attributed to the loss of physically adsorbed water, the next stage peak maximum at $386.3{ }^{\circ} \mathrm{C}$ is due to the decomposition.

The successful synthesis of metal sulfide nanoparticles offers an opportunity to examine their Photocatalytic activity, in order to study the effect of the UV light on the degradation of Copper sulfide and copper sulfide/graphene sheets nanocomposite.

The plot of absorption vs. wavelength at various times for the photodegradation of dye is represented in (Figure 5).

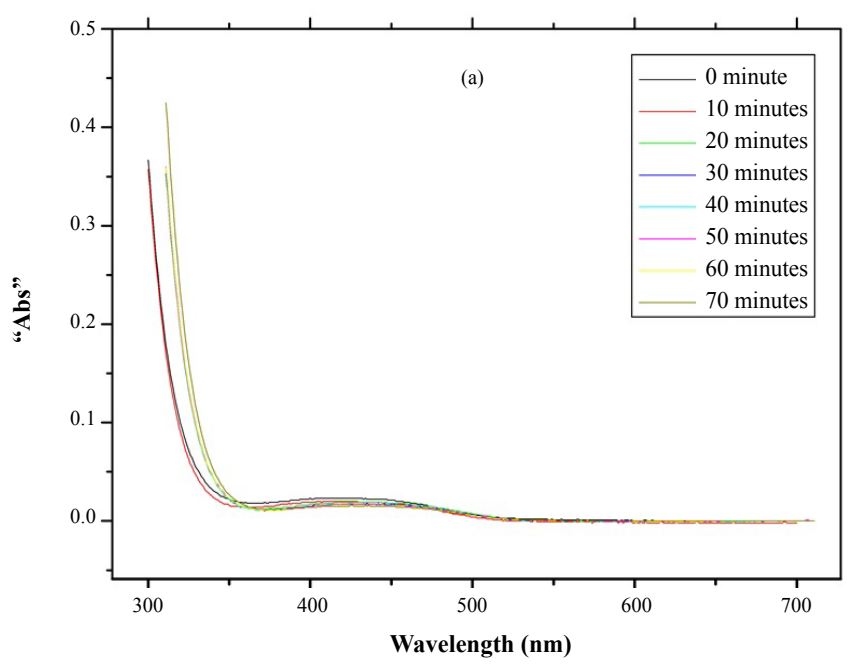

Figure 5 a shows the absorption peaks of Copper sulfide at different periods $(0,10,20,30,40,50$, 60 and 70) minutes. The copper sulfide/graphene sheets placed under $365 \mathrm{~nm}$ to $600 \mathrm{~nm}$ UV-V is irradiation, (Figure $5 \mathrm{~b}$ ) shows the absorption peaks at different periods $(0,15,30,45,60,75$ and 90) minutes. We can note that the highest absorption peaks in the region ( $362 \mathrm{~nm}-384.05 \mathrm{~nm}$ ), the absorption decrease with time, and at (420 nm-430 $\mathrm{nm}$ ) there is little increase $\sim(0.35)$, so the absorption decrease with time as shown in Figure $5 b$.

The higher photocatalytic property was probably caused by the smaller particle size and higher crystallinity. These results have shown that the prepared copper sulfide/graphene sheets (CuS/ Gs) photocatalysis higher than copper sulfide (CuS) photocatalysis in photocatalytic activity under UV light irradiation.

\section{Conclusion}

The synthesized copper sulfide nanoparticles were successfully prepared by co-precipitation method. The suggested reaction mechanism for the formation of CuS nanoparticles considers a combination of S-2 with $\mathrm{Cu}+2$ to form CuS. The XRD results show grain size of CuS ( $4 \mathrm{~nm}-10 \mathrm{~nm})$ with cubic structure and their structure compared by JCPDS data. TEM results revealed that these nanoparticles have grains with average sizes less than $10 \mathrm{~nm}$. Also, in this paper, we prepared CuS nanoparticles anchored onto Gs were prepared by hydrothermal method. The effect of nanocomposite graphene sheets on the composition of nanoparticles was in-

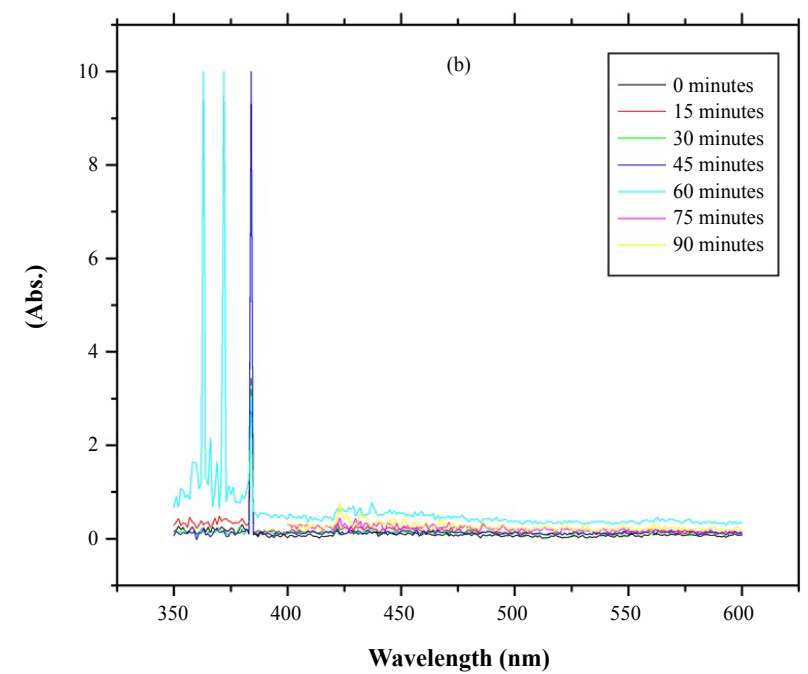

Figure 5: Photocatalysis of a) CuS and b) CuS/Gs. 
vestigated by EDX. The thermal resistance of CUS/ Gs is higher than the thermal resistance of CuS NPs, and thus enhanced the thermal properties of nanoparticles. The graphene sheet nanocomposite-based nanoparticles enhanced the photocatalytic properties. The synthesized CuS/Gs nanoparticles structure can be useful in photocatalytic application [17].

\section{Acknowledgments}

The work is supported by grant № 14-12-00275 of Russian Science Foundation and Saratov State University and Allahabad University, India.

\section{References}

1. Titipun Thongtem, Anukorn Phuruangrat, Somchai Thongtem (2010) Characterization of copper sulfide nanostructured spheres and nanotubes synthesized by microwave-assisted solvothermal method. Materials Letters 64: 136-139.

2. Mudi Xin, KunWei Li, Hao Wang (2009) Synthesis of CuS thin films by microwave assisted chemical bath deposition. Applied Surface Science 256: 1436-1442.

3. Lifei Chen, Yazhuo Shang, Honglai Liu, Ying Hua (2010) Synthesis of CuS nanocrystal in cationic gemini surfactant W/O microemulsion. Materials and Design 31: 1661-1665.

4. GW Yan, JH Huang, AP Jia, MF Luo (2009) Formation of CuS submicrotubes with quadrate cross section. Materials Research Bulletin 44: 1360-1365.

5. Fei Li, Jianfang $W u$, Qinghua Qin, Zhen Li, Xintang Huang (2010) Controllable synthesis, optical and photocatalytic properties of CuS nanomaterials with hierarchical structures. Powder Technology 198: 267-274.

6. Somchai Thongtem, Chaned Wichasilp, Titipun Thongtem (2009) Transient solid-state production of nanostructured CuS flowers. Materials Letters 63: 2409-2412.

7. LZ Pei, JF Wang, XX Tao, SB Wang, YP Dong, et al. (2011) Synthesis of CuS and $\mathrm{Cu}_{1.1} \mathrm{Fe}_{1.1} \mathrm{~S}_{2}$ crystals and their electrochemical properties. Materials Characterization 62: 354-359.
8. Anukorn Phuruangrat, $\mathrm{T}$ Thongtem, $\mathrm{S}$ Thongtem (2012) Controlling morphologies of $\mathrm{Bi}_{2} \mathrm{~S}_{3}$ nanostructures synthesized by glycolthermal method. Mater Lett 72: 104-106.

9. Titipun Thongtem, Anukorn Phuruangrat, Somchai Thongtem (2007) Synthesis and analysis of CuS with different morphologies using cyclic microwave irradiation. Journal of Materials Science 42: 9316-9323.

10.Titipun Thongtem, Anukorn Phuruangrat, Somchai Thongtem (2009) Formation of CuS with flower-like, hollow spherical, and tubular structures using the solvothermal-microwave process. Current Applied Physics 9: 195-200.

11.Y Zhang, Z-P Qiao, X-M Chen (2002) Microwave-assisted elemental-direct-reaction route to nanocrystalline copper sulfides $\mathrm{Cu}_{9} \mathrm{~S}_{8}$ and $\mathrm{Cu}_{7} \mathrm{~S}_{4}$. Journal of Solid State Chemistry 167: 249-253.

12.R Cordova, H Gomez, R Schrebler, P Cury, M Orellana, et al. (2002) Electrosynthesis and electrochemical characterization of a thin phase of $\mathrm{Cu}_{x} \mathrm{~S}(\mathrm{x} \rightarrow 2)$ on ITO electrode. Langmuir 18: 8647-8654.

13.Q Lu, F Gao, D Zhao (2002) One-step synthesis and assembly of copper sulfide nanoparticles to nanowires, nanotubes, and nanovesicles by a simple organic amine-assisted hydrothermal process. Nano Letters 2: 725-728.

14.IP Parkin (1996) Solid state metathesis reaction for metal borides, silicides, pnictides and chalcogenides: Ionic or elemental pathways. Chemical Society Reviews 25: 199-207.

15.E Godocıkova, P Balaz, E Gock, WS Choi, BS Kim (2006) Mechanochemical synthesis of the nanocrystalline semiconductors in an industrial mill. Powder Technology 164: 147-152.

16. R Nomura, K Miyawaki, T Toyosaki, H Matsuda (1996) Preparation of copper sulfide thin layers by a single source MOCVD process. Chemical Vapor Deposition 2: $174-178$.

17.Peng Huang, Zhiming Li, Hengyao Hu, Daxiang Cui (2010) Synthesis and Characterization of Bovine Serum Albumin-Conjugated Copper Sulfide Nanocomposites. Journal of Nanomaterials 2010: 1-6. 